\title{
Rev1 wbdR tagged vaccines against Brucella ovis
}

\author{
Beatriz Aragón-Aranda ${ }^{1 \dagger}$, María Jesús de Miguel²†, Estrella Martínez-Gómez , Amaia Zúñiga-Ripa', \\ Miriam Salvador-Bescós ${ }^{1}$, Ignacio Moriyón', Maite Iriarte', Pilar M. Muñoz ${ }^{2}$ and Raquel Conde-Álvarez ${ }^{1 *}$ (D)
}

\begin{abstract}
Sheep brucellosis is a worldwide extended disease caused by B. melitensis and B. ovis, two species respectively carrying smooth or rough lipopolysaccharide. Vaccine B. melitensis Rev1 is used against $B$. melitensis and B. ovis but induces an anti-smooth-lipopolysaccharide response interfering with $B$. melitensis serodiagnosis, which precludes its use against B. ovis where B. melitensis is absent. In mice, Rev1 deleted in wbkC (Brucella lipopolysaccharide formyltransferase) and carrying $w b d R$ (E. coli acetyl-transferase) triggered antibodies that could be differentiated from those evoked by wild-type strains, was comparatively attenuated and protected against B. ovis, suggesting its potential as a B. ovis vaccine.
\end{abstract}

\section{Introduction}

Brucellosis is one of the most common zoonoses worldwide, causing significant loss to animal production and severely affecting human health [1]. The etiological agents of brucellosis are Gram-negative bacteria of the genus Brucella. This genus includes several zoonotic species among which $B$. abortus preferentially infects cattle, $B$. suis swine and wild-life and $B$. melitensis goats and sheep [2]. Sheep can also be infected by B. ovis, a nonzoonotic species [2]. These species are smooth (S) or rough $(\mathrm{R})$, depending on the presence or absence respectively of O-polysaccharide (O-PS) chains in the outer membrane lipopolysaccharide (LPS). Whereas $B$. abortus, B. melitensis and B. suis carry a S-LPS, B. ovis is a naturally a $R$ species [2].

B. ovis infection causes genital lesions and reduced fertility constituting one of the most important causes of reproductive failure in sheep [3]. Animal vaccination is the most suitable method for controlling brucellosis in areas with moderate to high prevalence of the disease.

\footnotetext{
*Correspondence: rconde@unav.es

†Beatriz Aragón-Aranda and María Jesús de Miguel contributed equally to this work

${ }^{1}$ Instituto de Salud Tropical (ISTUN), Instituto de Investigación Sanitaria de Navarra (IdiSNA) and Dpto. de Microbiología y Parasitología,

Universidad de Navarra, c/Irunlarrea 1, 31008 Pamplona, Spain

Full list of author information is available at the end of the article
}

Currently, no specific vaccines against $B$. ovis infection are available, but the $\mathrm{S}$ live attenuated $B$. melitensis Rev1 vaccine, widely used for vaccination against ovine and caprine brucellosis caused by $B$. melitensis, is also effective against $B$. ovis [4]. However, Rev1 is virulent in humans, induces abortions when used in pregnant animals [4] and is resistant to streptomycin, an antibiotic of choice for brucellosis treatment [5]. While these problems can be solved by using appropriate vaccination strategies and biosafety precautions $[4,6], \operatorname{Rev} 1$ also induces a strong antibody response to the O-PS section of S-LPS [7] thus hampering differentiation between true infected and vaccinated animals (DIVA problem) in the routine diagnosis of $B$. melitensis. Because of this, Rev1 is banned in the countries where $B$. melitensis has been eradicated, resulting in a subsequent increase in $B$. ovis infections in sheep. Since $B$. ovis is naturally $\mathrm{R}$, some attempts to circumvent the problems associated with Rev1 vaccination in B. melitensis-free areas have been based on the use of $B$. ovis. Accordingly, some investigations have examined subcellular vaccines carrying $B$. ovis fractions rich in envelope components in lipid-muramyl dipeptide or nanoparticle adjuvants $[8,9]$. However, these formulations either provide less protection than Rev1 or become too costly. Also, B. ovis mutants in LPS core genes [10] have been explored with promising results. Similarly, a mutant in putative $A B C$ transporter encapsulated in alginate 
has been proposed as $B$. ovis vaccine $[11,12]$. Yet, industrial production of these vaccines would require solving the problem posed by $B$. ovis $\mathrm{CO}_{2}$-dependence [13] with the subsequent reassessment of their properties. Moreover the $\mathrm{ABC}$ transporter mutant requires encapsulation [12]. R mutants of S Brucella species (i.e., the so-called $R$ vaccines) are more easily produced and, as they lack the O-PS, are often assumed to solve the Rev1 DIVA problem. However, $R$ vaccines still interfere in S-LPS ELISA [14-16] because of the cross-reactivity with the core epitopes shared by the S and R-LPS or, in the wzm/wzt and related B. melitensis 115 spontaneous $\mathrm{R}$ mutants, presence of a cytoplasm O-PS precursor [1719]. Another approach was to delete protein BP26. However, whereas the BP26-deleted Rev1 provides protection against $B$. ovis, the ancillary BP26 ELISA lacks adequate diagnostic sensitivity $[20,21]$. More recently, a Rev1 construct expressing the green fluorescent protein as a tagging antigen has been proposed. However, this method requires the simultaneous injection of this protein and a booster injection to trigger antibody persisting in the ancillary ELISA-green fluorescent protein DIVA test [22].

The aim of this research is to investigate an alternative to circumvent the serological diagnosis problems caused by the Rev1 vaccine while keeping its good attenuation and protection characteristics. Our approach differs from those summarized above in that, instead of deleting $\mathrm{S}$ Brucella antigens or epitopes or introducing a foreign antigen, we have modified a Brucella immunodominant antigen. For this purpose, we applied to Rev1 the strategy proposed by Martínez-Gómez et al. [23] to modify the epitopic structure of Brucella S-LPS by substituting the $\mathrm{N}$-formyl-perosamine of the O-PS by $\mathrm{N}$-acetyl-perosamine. We present here the experiments carried out in the mouse model as a first step to investigate the validity of this approach.

\section{Materials and methods}

\section{Bacterial strains and growth conditions}

The bacterial strains and plasmids used are listed in Additional file 1. For construction of mutants, B. melitensis $16 \mathrm{M}$ and Rev1 strains were grown at $37^{\circ} \mathrm{C}$ in tryptic soy broth (TSB, Biomérieux, Marcy l'Etoile, France) or in this medium supplemented with agar (TSA, Pronadisa, Conda, Spain). B. ovis strains were grown at $37^{\circ} \mathrm{C}$ in TSB supplemented with $0.5 \%$ yeast extract (Pronadisa, Conda, Spain) and 5\% fetal bovine serum (TYSB-S) or this medium supplemented with agar (TYSA-S). For the studies in mice, vaccines and challenge strain were grown in Blood Agar Base (BAB, Oxoid) or BAB-S (supplemented with $5 \%$ fetal bovine serum). Where needed, media were supplemented with $5 \%$ sucrose (Sigma), diaminopimelic acid (DAP; $1 \mathrm{mM}$ ), 0.2\% activated charcoal (Sigma), kanamycin $(\mathrm{Km})$ at $50 \mu \mathrm{g} / \mathrm{mL}$, chloramphenicol $(\mathrm{Cm})$ at $20 \mu \mathrm{g} / \mathrm{mL}$, ampicillin (Amp) at $100 \mu \mathrm{g} / \mathrm{mL}$, polymyxin (Pmx) at $1.5 \mu \mathrm{g} / \mathrm{mL}$ or streptomycin (Strp) at $2.5 \mu \mathrm{g} /$ $\mathrm{mL}$. All strains were stored at $-80{ }^{\circ} \mathrm{C}$ in skimmed milk (Scharlau, Barcelona, Spain) or TYSB-7\% dimethylsulfoxide (DMSO).

\section{DNA manipulations}

Plasmid and chromosomal DNA were extracted with Q1Aprep ${ }^{\circledR}$ spin Miniprep Kit (Qiagen GmbH, Hilden, Germany) and Ultraclean Microbial DNA Isolation Kit (Mo Bio Laboratories), respectively. When needed, DNA was purified from agarose gels using a QIAquick Gel extraction kit (Qiagen). DNA sequencing was performed by "Servicio de Secuenciación del Centro de Investigación Médica Aplicada" (Pamplona, Spain). Primers were synthesized by Sigma-Genosys Ltd. (Haverhill, United Kingdom).

\section{Construction of mutants}

For the construction of Rev1::Tn7wbdRK $\mathrm{Km}^{\mathrm{R}}$ mutants, we used the plasmid pYRI-27 (pUC18R6KT-miniTn7T-KmP $w b d R)$ described in Martínez-Gómez et al. [23]. The acquisition of this vector by Brucella after tetra-parental mating with conjugative E. coli S17.1 $\lambda$ pir and E. coli HB101 (pRK2013) and E. coli SM10 $\lambda$ pir (pTNS2) was selected by $\mathrm{Km}$ and Pmx resistance. The correct insertion and orientation of the miniTn7 carrying $w b d R$ was examined by PCR as previously described [23].

To obtain a $w b d R$ construct with no $\mathrm{Km}$ resistance $(\operatorname{Rev} 1:: \operatorname{Tn} 7 w b d R)$, we used the plasmid pRCI-65 $\left(\right.$ pNPTS138 $\left.\mathrm{Cm}^{\mathrm{R}} \Delta K m\right)$ described in Martínez-Gómez et al. [23]. This suicide plasmid containing the $K m \mathrm{R}$ deletion allele was transformed into E. coli $\beta 2150$, a diaminopimelic acid (DAP) auxotrophic donor strain, to avoid the use of antibiotic during the conjugation process [24] and transferred into Rev1:: $\operatorname{Tn} 7 w b d R \mathrm{Km}^{\mathrm{R}}$ by conjugation. The integration of the suicide vector and disruption of the target gene were selected by $\mathrm{Km}$ sensitivity and confirmed by PCR using oligonucleotides $K m R$-F1 and $K m R$ R4 (Additional file 2).

Rev1:: $\operatorname{Tn} 7 w b d R \Delta w b k C$ was constructed in a similar way. The suicide plasmid pYRI-31 (pJQKm $\Delta w b k C)$ [23] was used to delete the $w b k C$ gene of Rev1::Tn7wbdR by allelic change. The resulting mutator plasmid was introduced in Rev1::Tn7wbdR by conjugation using the $E$. coli $\beta 2150$. The loss of the plasmid concomitant with the deletion of $w b k C$ gene was checked by PCR using oligonucleotides $w b k C$-F1 and $w b k C$-R4 (Additional file 2).

The strain $B$. ovis $\mathrm{PA}-\mathrm{KmR}$, used as challenge in mouse experiments (see below), was obtained using the 
modified miniTn7 site-specific integration vector technology $[25,26]$.

\section{Characterization of the mutants}

Mutants were characterized by the standard Brucella typing procedures described in Alton et al. [27]: colonial morphology, urease, susceptibility to thionine blue, fuchsine and safranine, acriflavine agglutination, crystal violet dye exclusion test ( $\mathrm{S} / \mathrm{R}$ colony morphology), agglutination with anti-A and anti-M monospecific sera, and sensitivity to phages. For co-agglutination, bacteria resuspended in $25 \mu \mathrm{L}$ of saline on a glass slide were mixed with an equal amount staphylococci sensitized with anti-Formyl-Acetyl, anti-Acetyl and anti-Formyl sera, as previously described [23].

\section{LPS extraction}

LPS from Rev1 and Rev1 $w b d R$ tagged was obtained by the proteinase-K sodium dodecyl sulfate (SDS) protocol $[28,29]$ with some modifications. Briefly, cells inactivated with phenol were suspended in $2 \%$ SDS- $60 \mathrm{mM}$ Tris- $\mathrm{HCl}$ buffer ( $\mathrm{pH}$ 6.8), heated at $100{ }^{\circ} \mathrm{C}$ for $10 \mathrm{~min}$ and treated with proteinase $\mathrm{K}(60 \mu \mathrm{L}$ of a $2.5 \mathrm{mg} / \mathrm{mL}$ stock per each $\mathrm{mL}$ of suspension, $3 \mathrm{~h}$ at $55^{\circ} \mathrm{C}$ ). This was followed by two consecutive precipitations with 3 volumes of methanol with $1 \%$ sodium acetate-saturated methanol at $-20{ }^{\circ} \mathrm{C}$. After centrifugation, pellets were resuspended by sonication in $3 \mathrm{~mL}$ of $60 \mathrm{mM} \mathrm{HCl}$-Tris (pH 6.8), digested with nucleases and treated again with proteinase $\mathrm{K}(3 \mathrm{~h}$ at $55^{\circ} \mathrm{C}$ ). After a third precipitation, the pellet containing LPS was recovered in $1 \mathrm{~mL}$ of distilled water and frozen.

\section{SDS-PAGE and Western blots}

Samples were mixed 1:1 with Sample buffer 2x (Bio-Rad), heated at $100{ }^{\circ} \mathrm{C}$ for $10 \mathrm{~min}$, and analyzed in $15 \%$ polyacrylamide gels in Tris-HCl-glycine and stained by the periodate-alkaline silver method [30]. For Western blots, LPS were analyzed in SDS-PAGE gel (12\% polyacrylamide) (see above) and electrotransferred onto nitrocellulose blotting sheets (Amersham- GE Healthcare Life Scientific, Germany; $0.45 \mu \mathrm{m}$ pore size). The polyclonal sera used were the following: Anti-S Brucella serum (polyclonal serum from a rabbit infected with B. melitensis $16 \mathrm{M}$ and bled at day 45), Formyl-Acetyl serum and Acetyl serum. These last two sera were obtained by the method described in Martínez-Gómez et al. [23].

\section{Multiplex PCR}

To discriminate between Rev1 $w b d R$ tagged strains and all known Brucella species and vaccine strains, we designed a pair of primers based on the $w b d R$ gene and added them to the 8 pairs of primers used in the
Bruce-ladder assay [31]. These new primers consisted of $5^{\prime}$-TGATGTTTTGGCAGGAAAGA-3' $(w b d R$ forward) and $5^{\prime}$-TAGCCCCAGGAGCAAATGTA- $3^{\prime}$ ( $w b d R$ reverse). We designed them with a Tm similar to that of the other primers used in the multiplex PCR and amplifying a band of $347 \mathrm{bp}$ that did not prevent the visualization of the other specific bands of species or vaccine strains.

\section{Studies in mice: virulence and protection}

Seven-week-old female BALB/C mice (ENVIGO, Harlan) were kept in cages in BSL-3 facilities (ES/31-2010000132) with water and food al libitum. Procedures were in accordance with the current European (directive 86/609/EEC) and Spanish (RD 53/2013) legislations, supervised by the corresponding Ethical Committee for Animal Experimentation and authorised by Aragón (reports No. 2014-20 and 2014-21) and Navarra (CEEA 045/12) Governments. For virulence assessment, 10-mice groups were inoculated intraperitoneally (IP) with $1 \times 10^{5}$ or $1 \times 10^{8}$ colony forming units (CFU) of the corresponding strain and mean $\mathrm{CFU}$ values per spleen were determined at $1(n=5)$ and $5(n=5)$ weeks after inoculation as described elsewhere [32]. To evaluate the protective efficacy of Rev1::Tn7wbdR $\Delta w b k C$ vaccine, 5-mice groups were vaccinated subcutaneously (SC) with two different doses $\left(1 \times 10^{5}\right.$ and $1 \times 10^{8} \mathrm{CFU} /$ mouse). Mice $(n=5)$ inoculated SC with the Rev1 reference vaccine $\left(1 \times 10^{5} \mathrm{CFU} / \mathrm{mouse}\right)$ or sterile Buffered Saline (BSS; $0.85 \% \mathrm{NaCl}, 0.1 \% \mathrm{KH}_{2} \mathrm{PO}_{4}, 0.2 \% \mathrm{~K}_{2} \mathrm{HPO}_{4}$; $\mathrm{pH}$ 6.85) were used as effective-vaccine and unvaccinated controls, respectively. Four weeks after vaccination, mice were challenged IP with $5 \times 10^{6} \mathrm{CFU} /$ mouse of $\mathrm{B}$. ovis $\mathrm{PA}-\mathrm{KmR}$ and the mean CFU/spleen values of this strain were determined 2 weeks after. Inocula were prepared by harvesting the $\mathrm{BAB}$ or $\mathrm{BAB}-\mathrm{S}$ grown bacteria in sterile BSS, adjusting spectrophotometrically $(600 \mathrm{~nm})$ the bacterial suspension and making proper serial tenfold dilutions. Mice were inoculated with $0.1 \mathrm{~mL}$ and doses were retrospectively assessed by plating inocula countable dilutions. Differentiation between challenge and vaccine strains was achieved by plating on BAB supplemented with streptomycin and BAB-S supplemented with $\mathrm{Km}$ (incubated in $\mathrm{CO}_{2}$ atmosphere). Results were expressed as the mean $\log _{10} \mathrm{CFU} /$ spleen $\pm \mathrm{SD}(n=5)$ of the corresponding mutant or challenge strain and units of protection were calculated by subtracting the mean $\log _{10} \mathrm{CFU}$ values of the vaccine group from those of unvaccinated controls. Statistical comparisons were made by one-way ANOVA and Fisher's Protected Least Significant Differences (PLSD) post hoc tests. The adequate virulence of B. ovis PA-KmR challenge strain was proved in a previous experiment in BALB/c mice by IP infection $\left(5 \times 10^{5}\right.$ 
$\mathrm{CFU} /$ mouse) and bacterial spleen counting (in BAB-S and BAB-S supplemented with $\mathrm{Km}) 3$ and 8 weeks later. This strain showed identical multiplication to that of $B$. ovis PA (not shown).

\section{Enzyme-linked immunosorbent assay (iELISA)}

Serum antibodies of mice inoculated with Rev1 (parental strain), Rev1::Tn7wbdR, Rev1::Tn7wbdR $\Delta w b k C$ and B. melitensis $16 \mathrm{M}$ (as control) were analyzed using an iELISA with wild-type ( $\mathrm{N}$-formyl-perosamine) or modified (N-acetyl-perosamine) S-LPS as antigens. For this, 96-well plates (Thermo Scientific, Waltham, MA, USA) were coated by overnight incubation at $4{ }^{\circ} \mathrm{C}$ with B. melitensis $16 \mathrm{M}$ or $\mathrm{Ba}:: \operatorname{Tn} 7 w b d R \Delta w b k C$ S-LPS at $2.5 \mu \mathrm{g} / \mathrm{mL}$ or $5 \mu \mathrm{g} / \mathrm{mL}$, respectively, in PBS. Plates were washed with PBS-0.05\% Tween 20 (and incubated with serial dilutions of sera at $37{ }^{\circ} \mathrm{C}$ for $30 \mathrm{~min}$. After washing, antibodies were detected with peroxidase-labeled protein G [19] and 2,2'-azino-bis (3-ethylbenzthiazoline-6-sulphonic acid) (ABTS) $-\mathrm{H}_{2} \mathrm{O}_{2}$. After 15 min, colorimetric reactions were read at $405 \mathrm{~nm}$ (Multiescanex, Thermo Scientific, Waltman, MA, USA). Results are expressed as optical density (O.D.) values of tested sera after subtracting the O.D. value of the negative control (blank well) in the same plate.

\section{Results}

Insertion of wbdR into Rev1 genome modifies the epitopic structure of the vaccine

To obtain a tagged Rev1 vaccine, we followed two strategies. First, we obtained Rev1::Tn7wbdR inserting $w b d R$ in the chromosome II of Rev1 vaccine using the previously described Tn7 methodology [8]. Second, we constructed a $w b d R$ tagged Rev1 lacking $\mathrm{N}$-formyl-perosamine in its O-PS (Rev1::Tn7wbdR $\Delta w b k C$ ) deleting $w b k C$ (the formyl transferase gene) from Rev1::Tn7wbdR. As expected, these constructs could be differentiated from Rev1, vaccine $B$. abortus S19, or representative strains of wild-type $B$. abortus, B. melitensis and B. ovis by PCR using the Multiplex-PCR combining Bruce-ladder and $w b d R$ specific primers (Figure 1).

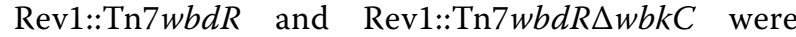
identical to the parental strain in colony morphology, growth, oxidase and urease tests and dye sensitivity

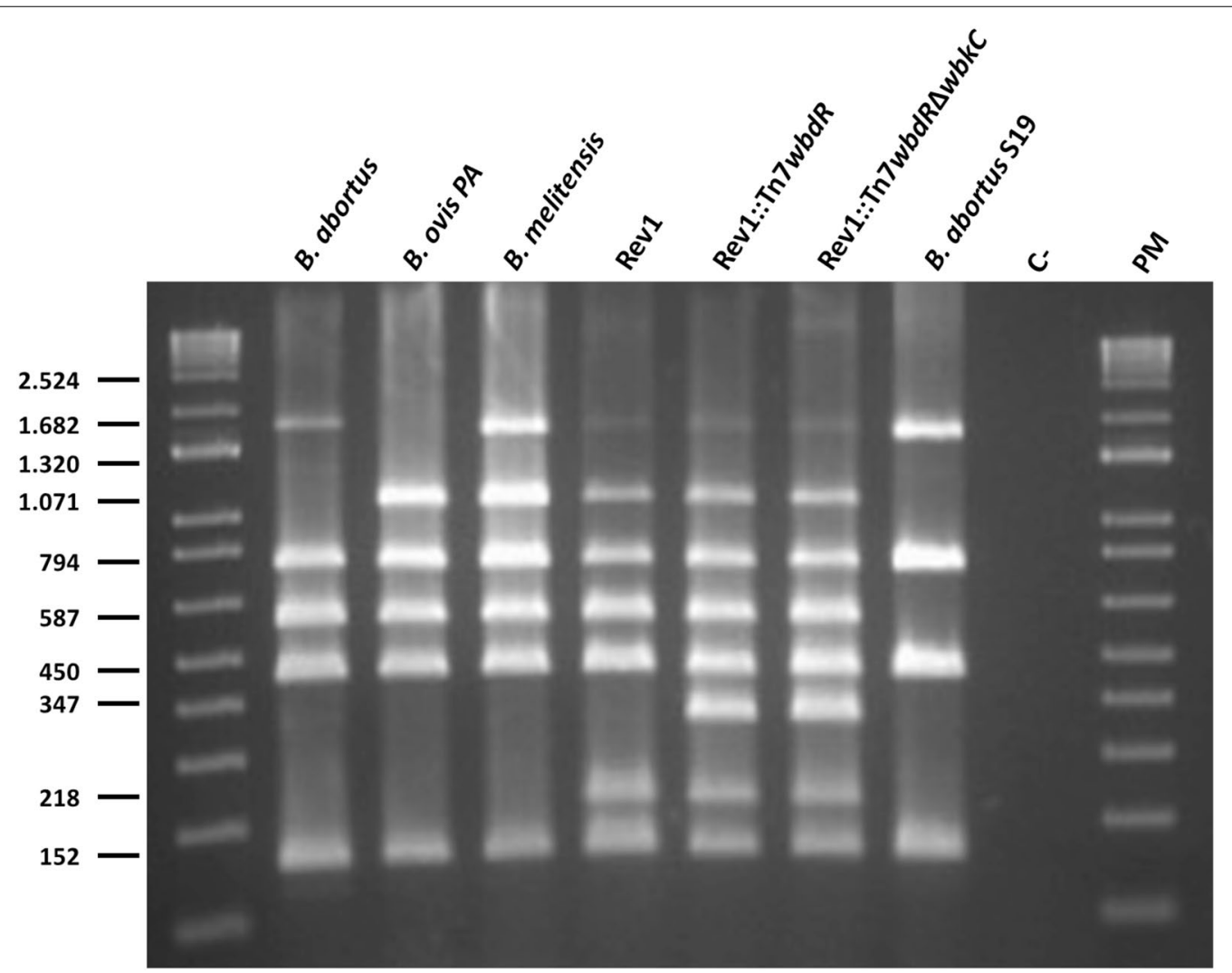

Figure 1 wbdR tagged vaccines can be identified by a modified multiplex PCR assay. Identification and differentiation of Brucella species, classical vaccines and Rev1 wbdR tagged vaccines by a modified Bruce-Ladder [31] that includes a new pair of primers based on the wbdR gene that amplify a band of 347 bp specific for tagged vaccines. $\mathrm{C}-\left(\mathrm{H}_{2} \mathrm{O}\right)$ and PM (1 Kb plus DNA ladder) used as molecular size marker. 
(Additional file 3). Consistent with previous results in virulent B. melitensis $16 \mathrm{M}$ [23], Rev1::Tn7wbdR and Rev1::Tn7wbdR $\Delta w b k C$ did not agglutinate with acriflavine and excluded crystal violet, properties characteristic of strains expressing S-LPS. On the other hand, both $w b d R$ tagged strains were sensitive to phage $\mathrm{R} / \mathrm{C}$, a phage specific for the O-PS-lacking R brucellae [27], a phenotype not observed previously on $w b d R$ constructs of virulent strains.

The LPS extracts of Rev1::Tn7wbdR and Rev1::Tn7wbdR $\Delta w b k C$ contained the typical R-LPS (i.e. low molecular weight) and S-LPS (higher molecular weight) fractions present in the parental strain (Figure 2). However, as observed before for their $w b d R$ tagged B. melitensis and B. abortus counterparts [23], the S-LPS fraction of the $w b d R$ constructs displayed an apparent average molecular weight lower than those of the parental strains (Figure 2A). To analyze possible epitopic changes associated with $w b d R$ tagging, whole cells and LPS extracts were tested by co-agglutination and Western blot, respectively, using immune sera specifically recognizing O-PS carrying only $\mathrm{N}$-formylperosamine (anti-Formyl), both $\mathrm{N}$-formyl-perosamine and N-acetyl-perosamine (anti-Formyl-Acetyl) or only $\mathrm{N}$-acetyl-perosamine (anti-Acetyl) [23]. Rev1::Tn7wbdR co-agglutinated with staphylococci sensitized with anti-Formyl-Acetyl, anti-Acetyl and anti-Formyl sera showing that its O-PS contained $\mathrm{N}$-acetyl and $\mathrm{N}$-formyl-perosamine. In contrast, Rev1::Tn7wbdR $\Delta w b k C$ did not co-agglutinate with anti-Formyl serum, while keeping the anti-Formyl-Acetyl and anti-Acetyl reactivity (Additional file 4). Western blots with proteinase-K extracted S-LPS (Figure 2B) confirmed the epitopic changes and demonstrated that the differences in apparent average molecular weight observed by SDS-PAGE (Figure 2A) corresponded in fact to O-PS heterogeneity.

\section{$w b d R$ tagging decreases the residual virulence of Rev 1} We vaccinated $\mathrm{BALB} / \mathrm{c}$ mice with $10^{5} \mathrm{CFU} /$ mouse (standard dose for Brucella S vaccines) of Rev1::Tn7wbdR, Rev1::Tn7wbdR $w w b k C$ and Rev1 and determined CFU numbers in spleen. One week post-infection, CFU/spleen values were significantly lower in mice infected with Rev1::Tn7wbdR or Rev1::Tn7wbdR $w w b k C$ than in those infected with Rev1 (Figure 3A). At week 5, though both Rev1::Tn7wbdR and Rev1::Tn7wbdR $\Delta w b k C$ also showed lower CFU, only the values of Rev1::Tn7wbdR $\Delta w b k C$ were statistically different (Figure $3 \mathrm{~A}$ ). However, attenuation with respect to Rev1 was clearly observed for both tagged vaccines at post-infection weeks 1 and 5 when the experiment was carried out with $10^{8} \mathrm{CFU} /$ mouse, the dose recommended for $\mathrm{R}$ vaccines (Figure $3 \mathrm{~B}$ ).

\section{Mice vaccinated with Rev1 wbdR constructs can be discriminated by ELISA}

We then analyzed by iELISA the sera of mice vaccinated with $10^{8} \mathrm{CFU}$ using first the S-LPS of Ba::Tn7wbdR $\Delta w b k C$ as antigen. As expected, and no matter the vaccine, no serum from these mice showed ELISA reactivity one week post-infection (not shown). At week 5, whereas most sera of mice inoculated with the $w b d R$ constructs showed some reactivity, sera of mice infected with Rev1 or B. melitensis did not react (Figure $4 \mathrm{~A}$ ). Interestingly, the reverse picture was obtained in the iELISA with wild-type S-LPS as antigen: whereas
A

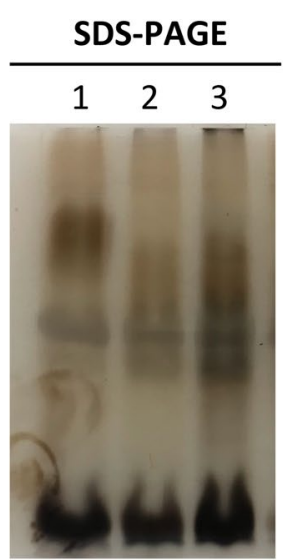

B

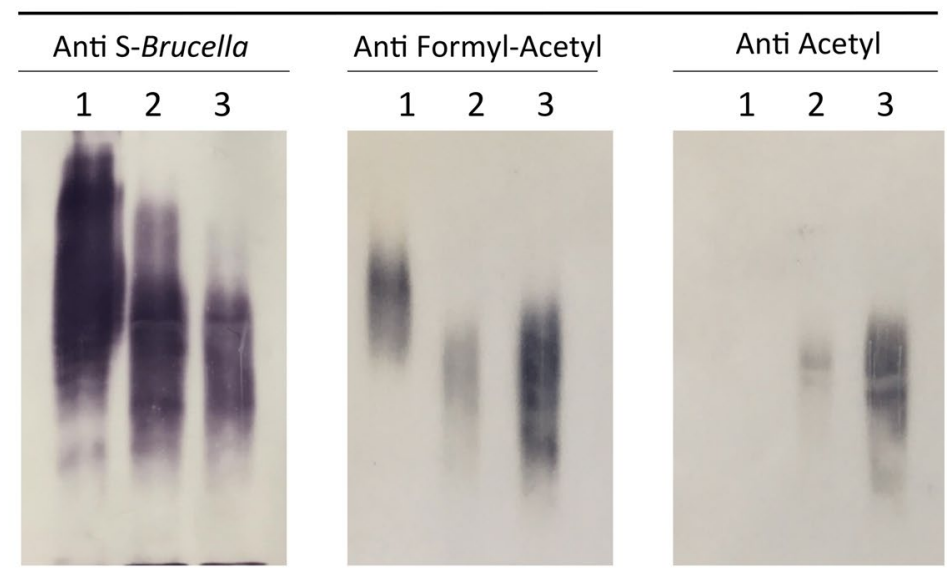

Figure 2 Insertion of $w b d R$ into Rev1 genome modifies the epitopic structure of the vaccine. A SDS-PAGE electrophoresis-silver staining and B Western blot analyses of SDS-proteinase K extracts of (1) Rev1, (2) Rev1::Tn7wbdR, and (3) Rev1::Tn7wbdRAwbkC. 
A

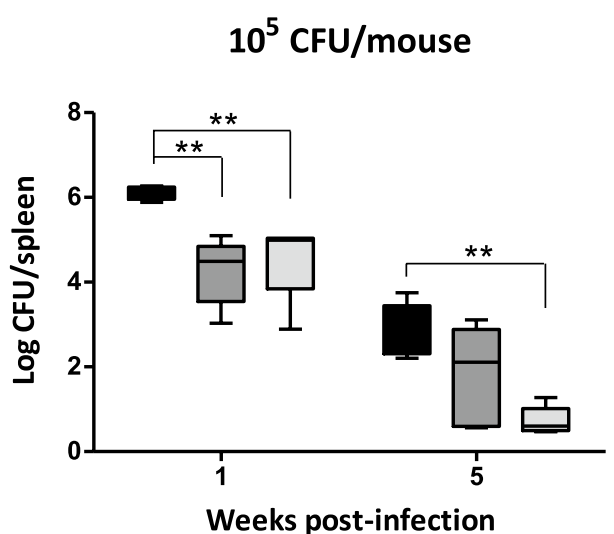

B

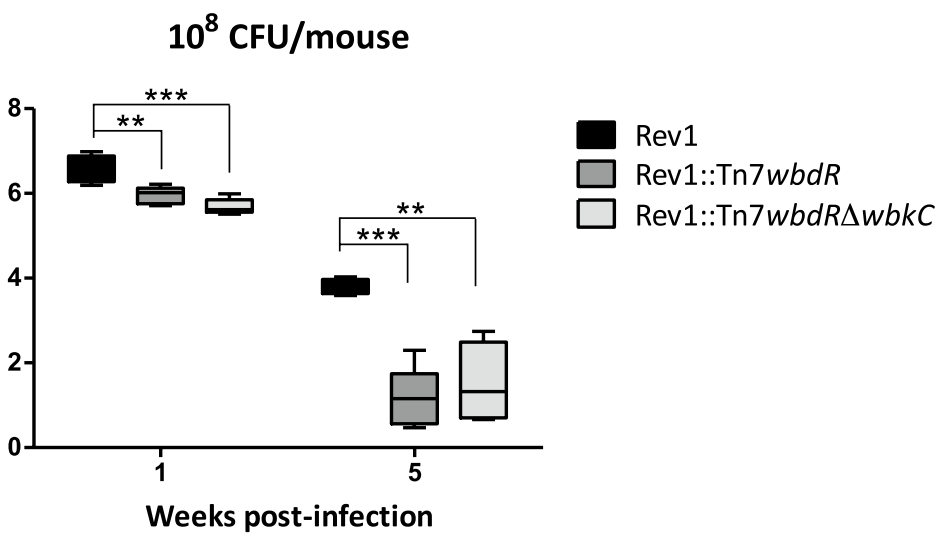

Figure $3 w \boldsymbol{b d R}$ tagging decreases the residual virulence of Rev1. BALB/C mice were inoculated with the indicated doses and CFU/spleen determined at post-infection weeks 1 and $5\left(^{*} p<0.05,{ }^{* *} p<0.01,{ }^{* * *} p<0.001\right)$.

\section{Antigen in iELISA:}

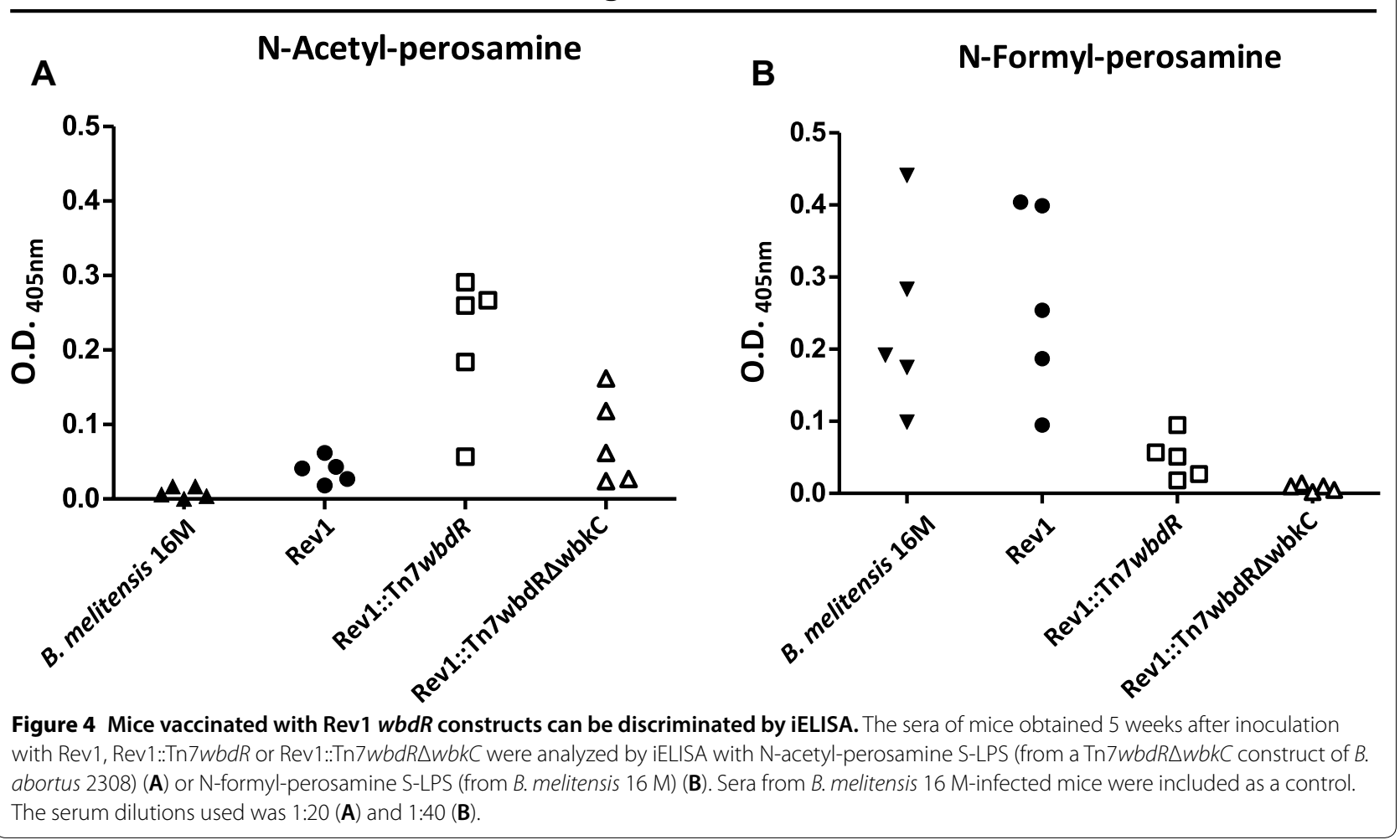

mice infected with Rev1 or B. melitensis developed reacting antibodies, sera of mice inoculated with $w b d R$ constructs displayed almost no reaction (Figure 4B) with small but consistently repeated (not shown) differences

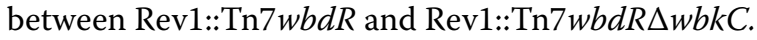

Rev1::Tn7wbdRAwbkC protects against $B$. ovis in the mouse model

Based on the ELISA results and on its lack of reactivity with the anti-Formyl serum (Additional file 4), we chose Rev1::Tn7wbdR $\Delta w b k C$ to study the efficacy of $w b d R$ tagged vaccines against $B$. ovis. As shown in Table 1, this vaccine conferred protection at the two 
Table 1 Protection induced by Rev1::Tn7wbdRAwbkC against $B$. ovis

\begin{tabular}{|c|c|c|c|}
\hline \multirow[t]{2}{*}{ Vaccine (dose) } & \multicolumn{2}{|c|}{$\begin{array}{l}\text { Mean } \pm \text { SD of } \log _{10} \text { CFU } \\
\text { in spleen of: }\end{array}$} & \multirow[t]{2}{*}{$\begin{array}{l}\text { Units } \\
\text { of protection }\end{array}$} \\
\hline & B. ovis & Vaccine & \\
\hline $\operatorname{Rev1}\left(10^{5}\right)$ & $2.45 \pm 1.35^{\mathrm{a}}$ & $2.82 \pm 0.99$ & 3.81 \\
\hline $\operatorname{Rev} 1: \operatorname{Tn} 7 w b d R \Delta w b k C\left(10^{5}\right)$ & $4.47 \pm 1.40$ & $0.69 \pm 0.09^{c}$ & 1.79 \\
\hline $\operatorname{Rev} 1: \operatorname{Tn} 7 w b d R \Delta w b k C\left(10^{8}\right)$ & $2.16 \pm 0.95^{\mathrm{a}, \mathrm{b}}$ & $0.90 \pm 0.53^{c}$ & 4.10 \\
\hline PBS (unvaccinated) & $6.26 \pm 0.16$ & & 0.00 \\
\hline
\end{tabular}

Statistical comparison ( $n=5$ ) of mean $\log _{10}$ B. ovis CFU/spleen: ${ }^{a} p<0.001$ vs. PBS (unvaccinated), ${ }^{b} p>0.05$ vs. Rev1 and $\log _{10}$ vaccine CFU/spleen ${ }^{c} p<0.01$ vs. Rev1.

doses tested. However, only the $10^{8} \mathrm{CFU} /$ mouse dose (used for rough vaccines in the mouse model) conferred a protection similar to Rev1 at the standard dose (Table 1). Interestingly, even at this higher dose, the vaccine $\mathrm{CFU}$ remaining in the spleens was markedly lower than those of $\operatorname{Rev1}(p<0.01)$.

\section{Discussion}

The results presented in this work demonstrate that the O-PS of vaccine Rev1 can be antigenically tagged by genomic insertion of $w b d R$, the acetyltransferase gene involved in the synthesis of the O-PS repeating unit of E. coli O157:H7. Using the mouse model, we studied the protective capacity of $w b d R$ tagged Rev1 against $B$. ovis and tested an ancillary ELISA DIVA test. In previous and preliminary experiments, we examined whether $w b d R$ tagged brucellae $(w b d R \Delta w b k C)$ could be used as antigen in a simple test similar to the Rose Bengal test for brucellosis. However, we observed that the modified brucellae had a marked tendency to autoagglutinate, a feature that is reminiscent of the autoagglutination typical of R brucellae [16] and shows profound cell surface modifications. For this reason, we ruled out the agglutination test and applied an iELISA using the LPS purified from a $w b d R \Delta w b k C$ construct [23] adjusted to minimize any cross-reactivity between acety-tagged and native O-PS. In this assay, we observed differences between the antibodies triggered by $w b d R$ tagged vaccines when compared with those triggered by Rev1; whereas sera of mice vaccinated with Rev1::Tn7wbdR or Rev1::Tn7wbdR $\Delta w b k C$ showed moderate reaction, sera corresponding to the Rev1 vaccinated mice did not react. Moreover, when the iELISA with wild-type S-LPS was used, contrary to the diagnostically interfering reaction showed by Rev1, the sera of mice inoculated with $w b d R$ constructs displayed almost no reaction. In a previous work, we observed that the LPS of Brucella crossreacting $E$. hermanii serotypes reacts strongly with sera from infected cattle [21,33] which are known to contain an overwhelming majority of antibodies of overlapping $C$ specificities [34]. The O-PS of these E. hermanii strains contains $\mathrm{N}$-acetylated perosamine in an $\alpha(1-3)$ and $\alpha(1-2)$ linkage frequency and arrangement different from those in Rev1 (i.e. B. melitensis biovar 1) and cross-react with Brucella $\mathrm{M}$ monoclonal antibodies in gel immunoprecipitation [33]. The O-PS in the $w b d R \Delta w b k C$ constructs does not react in ELISA with $\mathrm{C} / \mathrm{Y}-\mathrm{A}=\mathrm{M}$ or al $\mathrm{C} / \mathrm{Y}-\mathrm{A}>\mathrm{M}$ in ELISA [23]. These data suggest epitopic differences that, in addition to differences in the intensity of the stimulus in an experimental infection in mice and a natural infection in cattle, in the subsequent different avidity of the antibodies and in the standardization of the ELISA, could account for these apparently contradictory results. Our results suggest that the use of $w b d R$ tagged Rev1 and its ancillary ELISA might be a suitable strategy to solve the DIVA problem associated with the unmodified vaccine, but it is clear that experiments in sheep are necessary for a definite assessment.

In keeping with previous studies in virulent brucellae [23], the most profound modification was obtained in Rev1::Tn7wbdR $\Delta w b k C$ and, accordingly, we tested its protective capacity in the mouse model. We observed that a $10^{8} \mathrm{CFU} /$ mouse dose was necessary to reach the protection levels obtained with Rev1 at $10^{5} \mathrm{CFU} /$ mouse. The recommended dose for brucellosis $\mathrm{R}$ vaccines is also $10^{8} \mathrm{CFU} /$ mouse [35] and, like the autoagglutination commented above this suggests surface similarities between the Rev1::Tn7wbdR $\Delta w b k C$ construct and $\mathrm{R}$ brucellae. The lack of O-PS in the latter increases the exposure of lipid A-core and Omp charged groups and it has been postulated that the OP-S acts as a negative modulator of unspecific interactions of these bacteria with host cells, thus favoring invasion for specific routes [18]. On these bases, it can be hypothesized that either the N-acetylperosamine O-PS cannot replace the $\mathrm{N}$-formyl-perosamine with regards to surface physicochemical properties and/or that the shorter O-PS of Rev1::Tn7wbdR $\Delta w b k C$ constructs does not provide enough steric hindrance to the Omps and the lipid A-core section, as suggested by its sensitivity to the $\mathrm{R} / \mathrm{C}$ phage. These surface changes may account for our observation that, when tested for the residual virulence (Figure 3), we found that the CFU counts/spleen of Rev1::Tn7wbdR $\Delta w b k C$ were lower than those of Rev1 even at a dose one thousand times higher, a result in agreement with the low numbers of CFU remaining in the spleens in the vaccination experiments (Table 1). Taken together, these results in mice suggest that Rev1::Tn7wbdR $\Delta w b k C$ could be not only a DIVA vaccine but also a vaccine with reduced residual virulence in the host. Research is in progress to evaluate 
serological response and protective capacity against $B$. ovis of Rev1::Tn7wbdR $\Delta w b k C$ in sheep.

\section{Supplementary information}

Supplementary information accompanies this paper at https://doi. org/10.1186/s13567-019-0714-3.

\section{Additional file 1. Bacterial strains and plasmids.}

Additional file 2. Primers.

Additional file 3. Differential characteristics of species of the genus Brucella and mutants.

Additional file 4. Insertion of $w b d R$ into Rev1 genome modifies the epitopic structure of the vaccine.

\section{Acknowledgements}

We thank A. Delgado-López for excellent technical assistance. We are also grateful to an anonymous reviewer for a helpful discussion of the results.

\section{Authors' contributions}

RC-A, PM and IM conceived and coordinated the study. MI supervised the genomic studies. BA-A, MJdM, EM-G, AZ-R and MS-B performed experiments. RC-A, BA-A, PM, and IM wrote the manuscript. All authors analyzed the results and approved the final version of the manuscript. RC-A, AZ-R, MI and IM are inventors of patent EP15201717.4 covering potential uses of $w b d R$ constructs. All authors read and approved the final manuscript.

\section{Funding}

This research was supported by the Institute for Tropical Health funders (Obra Social la CAIXA-LCF/PR/PR13/11080005- and Fundación Caja Navarra, Fundación María Francisca de Roviralta, Ubesol and Inversiones Garcilaso de la Vega S.L.) and grants MINECO (AGL2014-58795-C4-1-R). B.A.A. is the recipient of the Ph.D. Fellowship BES-2015-075609 funded by the MINECO.

\section{Ethics approval and consent to participate}

Studies in mice were done in accordance with the current European (directive 86/609/EEC) and Spanish (RD 53/2013) legislations, supervised by both Ethical Committee for Animal Experimentation of CITA and Animal Welfare Committee of the University of Navarra and authorised by Aragón (reports No. 2014-20 and 2014-21) and Navarra (CEEA 045/12) Governments.

\section{Competing interests}

The authors declare that they have no competing interests.

\section{Author details}

1 Instituto de Salud Tropical (ISTUN), Instituto de Investigación Sanitaria de Navarra (IdiSNA) and Dpto. de Microbiología y Parasitología, Universidad de Navarra, c/Irunlarrea 1, 31008 Pamplona, Spain. ${ }^{2}$ Unidad de Producción y Sanidad Animal, Instituto Agroalimentario de Aragón-IA2 (CITA-Universidad de Zaragoza), Av. Montañana 930, 50059 Saragossa, Spain.

Received: 5 July 2019 Accepted: 23 October 2019

Published online: 15 November 2019

\section{References}

1. McDermott JJ, Grace D, Zinsstag J (2013) Economics of brucellosis impact and control in low-income countries. Rev Sci Tech 32:249-261

2. Moreno E (2014) Retrospective and prospective perspectives on zoonotic brucellosis. Front Microbiol 5:213

3. Blasco JM (1990) Brucella ovis. In: Nielsen KH, Duncan JR (eds) In: Animal brucellosis. CRC Press, Boca Raton, pp 352-378

4. Blasco JM, Molina-Flores B (2011) Control and eradication of Brucella melitensis infection in sheep and goats. Vet Clin North Am Food Anim Pract 27:95-104
5. Ariza J, Bosilkovski M, Cascio A, Colmenero JD, Corbel MJ, Falagas ME, Memish A, Roushan MRH, Rubinstein E, Sipsas NV, Solera J, Young EJ, Pappas G, International Society of Chemotherapy; Institute of Continuing Medical Education of loannina (2007) Perspectives for the treatment of brucellosis in the 21st century: rhe loannina recommendations. PLoS Med 4:e317

6. OIE (2018) Manual of Diagnostic Tests and Vaccines for Terrestrial Animals. World Organ Anim Heal. Brucellosis pp:355-398

7. Marín CM, Moreno E, Moriyón I, Díaz R, Blasco JM (1999) Performance of competitive and indirect enzyme-linked immunosorbent assays, gel immunoprecipitation with native hapten polysaccharide, and standard serological tests in diagnosis of sheep brucellosis. Clin Diagn Lab Immunol 6:269-272

8. Blasco JM, Gamazo C, Winter AJ, Jiménez de Bagüés MP, Marín C, Barberán M, Moriyón I, Alonso-Urmeneta B, Díaz R (1993) Evaluation of whole cell and subcellular vaccines against Brucella ovis in rams. Vet Immunol Immunopathol 37:257-270

9. Da Costa Martins R, Irache JM, Blasco JM, Muñoz MP, Marín CM, Grilló MJ, De Miguel MJ, Barberán M, Gamazo C (2010) Evaluation of particulate acellular vaccines against Brucella ovis infection in rams. Vaccine 28:3038-3046

10. Soler-Lloréns P, Gil-Ramírez Y, Zabalza-Baranguá A, Iriarte M, Conde-Álvarez R, Zúñiga-Ripa A, San Román B, Zygmunt MS, Vizcaíno N, Cloeckaert A, Grilló MJ, Moriyón I, López-Goñi I (2014) Mutants in the lipopolysaccharide of Brucella ovis are attenuated and protect against B ovis infection in mice. Vet Res 45:72

11. Silva APC, Macêdo AA, Silva TMA, Ximenes LCA, Brandão HM, Paixão TA, Santos $L$ (2015) Protection provided by an encapsulated live attenuated $\triangle$ abcBA strain of Brucella ovis against experimental challenge in a murine model. Clin Vaccine Immunol 22:789-797

12. Silva APC, Macêdo AA, Costa LF, Rocha CE, Garcia LNN, Farias JRD, Gomes PPR, Teixeira GC, Fonseca KWJ, Maia ARF, Neves GG, Romão EL, Silva TMA, Mol JPS, Oliveira RM, Araújo MSS, Nascimento EF, Martins-Filho OA, Brandão HM, Paixão TA, Santoset RL (2015) Encapsulated Brucella ovis lacking a putative ATP-binding cassette transporter $(\triangle \mathrm{abcBA})$ protects against wild type Brucella ovis in rams. PLoS One 10:e0136865

13. Pérez-Etayo L, de Miguel MJ, Conde-Álvarez R, Muñoz PM, Khames M, Iriarte M, Moriyón I, Zúñiga-Ripa A (2018) The CO2-dependence of Brucella ovis and Brucella abortus biovars is caused by defective carbonic anhydrases. Vet Res 49:85

14. Barrio MB, Grilló MJ, Muñoz PM, Jacques I, González D, de Miguel MJ, Marín CM, Barberán M, Letesson JJ, Gorvel JP, Moriyón I, Blasco JM, Zygmunt MS (2009) Rough mutants defective in core and O-polysaccharide synthesis and export induce antibodies reacting in an indirect ELISA with smooth lipopolysaccharide and are less effective than Rev 1 vaccine against Brucella melitensis infection of sheep. Vaccine 27:1741-1749

15. Gusi AM, Bertu WJ, Jesús de Miguel M, Dieste-Pérez L, Smits HL, Ocholi RA, Blasco JM, Moriyón I, Muñoz PM (2019) Comparative performance of lateral flow immunochromatography, iELISA and Rose Bengal tests for the diagnosis of cattle, sheep, goat and swine brucellosis. PLoS Negl Trop Dis 13:e0007509

16. Ducrotoy MJ, Conde-Álvarez R, Blasco JM, Moriyón I (2016) A review of the basis of the immunological diagnosis of ruminant brucellosis. Vet Immunol Immunopathol 171:81-102

17. Godfroid F, Cloeckaert A, Taminiau B, Danese I, Tibor A, de Bolle X, Mertens P, Letesson JJ (2000) Genetic organisation of the lipopolysaccharide O-antigen biosynthesis region of Brucella melitensis $16 \mathrm{M}$ (wbk). Res Microbiol 151:655-668

18. González D, Grilló MJ, De Miguel MJ, Ali T, Arce-gorvel V, Delrue RM, Conde-Alvarez R, Muñoz PM, López-Goñi I, Iriarte M, Marín CM, Weintraub A, Widmalm G, Zygmunt M, Letesson JJ, Gorvel JP, Blasco JM, Moriyón I (2008) Brucellosis vaccines: assessment of Brucella melitensis lipopolysaccharide rough mutants defective in core and O-polysaccharide synthesis and export. PLOS ONE 3:e2760

19. Pérez-Sancho M, Adone R, García-Seco T, Tarantino M, Diez-Guerrier A, Drumo R, Francia M, Domínguez L, Pasquali P, Álvarez J (2014) Evaluation of the immunogenicity and safety of Brucella melitensis B1 15 vaccination in pregnant sheep. Vaccine 32:1877-1881

20. Grilló MJ, Marín CM, Barberán M, de Miguel MJ, Laroucau K, Jacques I, Blasco JM (2009) Efficacy of bp26 and bp26/omp31 B. melitensis Rev. 1 deletion mutants against Brucella ovis in rams. Vaccine. 27:187-191 
21. Muñoz PM, Marin CM, Monreal D, Gonzalez D, Garin-Bastuji B, Diaz R, Mainar-Jaime RC, Moriyon I, Blasco JM (2005) Efficacy of several serological tests and antigens for diagnosis of bovine brucellosis in the presence of false-positive serological results due to Yersinia enterocolitica 0:9. Clin Vaccine Immunol 12:141-151

22. Zabalza-Baranguá A, San-Román B, Chacón-Díaz C, de Miguel M-J, Muñoz P-M, Iriarte M, Blasco JM, Grilló MJ (2019) GFP tagging of Brucella melitensis Rev1 allows the identification of vaccinated sheep. Transbound Emerg Dis 66:505-516

23. Martínez-Gómez E, Ståhle J, Gil-Ramírez Y, Zúñiga-Ripa A, Zaccheus M, Moriyón I, Iriarte M, Widmalm G, Conde-Álvarez R (2018) genomic insertion of a heterologous acetyltransferase generates a new lipopolysaccharide antigenic structure in Brucella abortus and Brucella melitensis. Front Microbiol 9:1092

24. Allard N, Garneau D, Poulin-Laprade D, Burrus V, Brzezinski R, Roy S (2015) A diaminopimelic acid auxotrophic Escherichia coli donor provides improved counterselection following intergeneric conjugation with actinomycetes. Can J Microbiol 61:565-574

25. Choi K-H, Gaynor JB, White KG, Lopez C, Bosio CM, Karkhoff-Schweizer RR, Schweizer HP (2005) A Tn7-based broad-range bacterial cloning and expression system. Nat Methods 2:443-448

26. Llobet E, March C, Gimenez P, Bengoechea JA (2009) Klebsiella pneumoniae OmpA confers resistance to antimicrobial peptides. Antimicrob Agents Chemother 53:298-302

27. Alton GG, Jones LM, Angus RD, Verger J-M (1988) Techniques for the brucellosis laboratory. INRA, Paris

28. Dubray G, Limet J (1987) Evidence of heterogeneity of lipopolysaccharides among Brucella biovars in relation to $A$ and $M$ specificities. Ann Inst Pasteur Microbiol 138:27-37

29. Garin Bastuji B, Bowden RA, Dubray G, Limet JN (1990) Sodium dodecyl sulfate-polyacrylamide gel electrophoresis and immunoblotting analysis of smooth-lipopolysaccharide heterogeneity among Brucella biovars related to A and M specificities. J Clin Microbiol 28:2169-2174

30. Tsai C-M, Frasch CE (1982) A sensitive silver stain for detecting lipopolysaccharides in polyacrylamide gels. Anal Biochem 119:115-119

31. López-Goñi I, García-Yoldi D, Marín CM, de Miguel MJ, Barquero-Calvo E, Guzmán-Verri C, Albert D, Garin-Bastuji B (2011) New Bruce-ladder multiplex PCR assay for the biovar typing of Brucella suis and the discrimination of Brucella suis and Brucella canis. Vet Microbiol 154:152-155

32. Grilló MJ, Manterola L, de Miquel MJ, Muñoz PM, Blasco JM, Moriyón I, López-Goñi I (2006) Increases of efficacy as vaccine against Brucella abortus infection in mice by simultaneous inoculation with avirulent smooth bvrS/bvrR and rough wbkA mutants. Vaccine 24:2910-2916

33. Perry MB, Bundle DR (1990) Antigenic relationships of the lipopolysaccharides of Escherichia hermannii strains with those of Escherichia coli O157:H7, Brucella melitensis, and Brucella abortus. Infect Immun 58:1391-1395

34. Alonso-Urmeneta B, Marín C, Aragón V, Blasco JM, Díaz R, Moriyón I (1998) Evaluation of lipopolysaccharides and polysaccharides of different epitopic structures in the indirect enzyme-linked immunosorbent assay for diagnosis of brucellosis in small ruminants and cattle. Clin Diagn Lab Imunol 5:749-754

35. Moriyón I, Grilló MJ, Monreal D, González D, Marín C, López-Goñi I, Moreno E, Blasco JM (2004) Rough vaccines in animal brucellosis: structural and genetic basis and present status. Vet Res 35:1-38

\section{Publisher's Note}

Springer Nature remains neutral with regard to jurisdictional claims in published maps and institutional affiliations.
Ready to submit your research? Choose BMC and benefit from:

- fast, convenient online submission

- thorough peer review by experienced researchers in your field

- rapid publication on acceptance

- support for research data, including large and complex data types

- gold Open Access which fosters wider collaboration and increased citations

- maximum visibility for your research: over 100M website views per year

At BMC, research is always in progress.

Learn more biomedcentral.com/submissions 